\title{
A!
}

This is an electronic reprint of the original article.

This reprint may differ from the original in pagination and typographic detail.

Jääskeläinen, Anna; Colombo, Luciano; Nieminen, Risto

\section{Silicon self-diffusion constants by tight-binding molecular dynamics}

Published in:

Physical Review B

DOI:

10.1103/PhysRevB.64.233203

Published: 15/12/2001

Document Version

Publisher's PDF, also known as Version of record

Please cite the original version:

Jääskeläinen, A., Colombo, L., \& Nieminen, R. (2001). Silicon self-diffusion constants by tight-binding molecular dynamics. Physical Review B, 64(23), 1-3. [233203]. https://doi.org/10.1103/PhysRevB.64.233203

This material is protected by copyright and other intellectual property rights, and duplication or sale of all or part of any of the repository collections is not permitted, except that material may be duplicated by you for your research use or educational purposes in electronic or print form. You must obtain permission for any other use. Electronic or print copies may not be offered, whether for sale or otherwise to anyone who is not an authorised user. 


\title{
Silicon self-diffusion constants by tight-binding molecular dynamics
}

\author{
Anna Jääkeläinen, ${ }^{1}$ Luciano Colombo, ${ }^{2, *}$ and Risto Nieminen ${ }^{1}$ \\ ${ }^{1}$ Laboratory of Physics, Helsinki University of Technology, P.O. Box 1100, FIN-02015 HUT, Finland \\ ${ }^{2}$ Istituto Nazionale per la Fisica della Materia and Department of Physics, University of Cagliari, Cittadella Universitaria, \\ I-09042 Monserrato (CA), Italy
}

(Received 16 May 2001; revised manuscript received 19 July 2001; published 29 November 2001)

\begin{abstract}
The thermodynamic integration method has been incorporated into the tight-binding molecular-dynamics scheme to compute formation free energies of native point defects in bulk silicon. By combining previous simulated diffusivity data with present free-energy estimates, we present a thorough quantum-mechanical picture of self-diffusion in silicon that is both consistent with the state-of-the-art experimental data and able to predict separately the vacancy and self-interstitial contributions.
\end{abstract}

DOI: 10.1103/PhysRevB.64.233203

Self-diffusion in silicon has been the subject of intense research since it rules many fundamental materials phenomena, such as equilibrium and nonequilibrium properties of native defects and processes of matter transport. It also underlies the microstructural evolution of bulk silicon under many technologically-relevant processes, such as Czochralski crystal growth or ion implantation. It is nowadays well established that the self-diffusion coefficient $D_{S D}(T)$ follows an Arrhenius equation

$$
D_{S D}(T)=D^{0} \exp \left(-E^{a} / k_{B} T\right),
$$

over a wide range of temperatures. ${ }^{1}$ Recent experimental measurements of self-diffusion in isotope heterostructures ${ }^{2}$ have shown a temperature dependence accurately described over many orders of magnitude by an activation energy of migration $E^{a}=4.75 \pm 0.04 \mathrm{eV}$ and by a preexponential factor $D^{0}=530_{-170}^{+250} \mathrm{~cm}^{2} \mathrm{~s}^{-1}$.

Although the global picture is clear, there is still uncertainty about the relative contributions to $D_{S D}(T)$ due to the various elementary mechanisms of diffusion. As a matter of fact, self-diffusion is influenced by vacancy $(V)$ and selfinterstitial $(I)$ migration, as well as by the direct exchange of two $\mathrm{Si}$ atoms, occurring between two neighboring lattice sites. ${ }^{3}$ This latter mechanism, however, is usually disregarded since no experimental evidence has been found for it, $^{2}$ and its contribution to $D_{S D}(T)$ has been theoretically predicted to be negligibly small. ${ }^{4}$ Accordingly, the selfdiffusion coefficient can be cast in the form ${ }^{2}$

$$
D_{S D}(T)=f_{I} C_{I}^{e q}(T) d_{I}(T)+f_{V} C_{V}^{e q}(T) d_{V}(T),
$$

where $C_{I}^{e q}(T)$ and $C_{V}^{e q}(T)$ represent, respectively, the (normalized) equilibrium concentration of $V$ 's and $I$ 's defects, while $d_{I}(T)$ and $d_{V}(T)$ indicate the corresponding diffusivities. The $f_{I}$ and $f_{V}$ terms represent the autocorrelation factors for native defect migration trajectories and are well known for the diamond lattice. ${ }^{5}$ The open problem, therefore, consists of providing separately an estimation of $C_{I}^{e q}, C_{V}^{e q}, d_{I}, d_{V}$ and their explicit dependences upon temperature.

By means of metal diffusion experiments ${ }^{6}$ it has been possible to characterize the $I$-contribution to self-diffusion as

$$
C_{I}^{e q}(T) d_{I}(T)=2980 \exp \left(-4.95 \mathrm{eV} / k_{B} T\right) \quad \mathrm{cm}^{2} \mathrm{~s}^{-1},
$$

PACS number(s): 66.30.Hs, 61.72.Ji, 61.72.Bb

which, combined with the above result for the total $D_{S D}(T)$, allows to evaluate the $V$ contribution as ${ }^{2}$

$$
C_{V}^{e q}(T) d_{V}(T)=0.92 \exp \left(-4.14 \mathrm{eV} / k_{B} T\right) \mathrm{cm}^{2} \mathrm{~s}^{-1} .
$$

Other experimental data ${ }^{7}$ have been published, according to which the above diffusion constants should be

$$
C_{I}^{e q}(T) d_{I}(T)=914 \exp \left(-4.84 \mathrm{eV} / k_{B} T\right) \mathrm{cm}^{2} \mathrm{~s}^{-1},
$$

and

$$
C_{V}^{e q}(T) d_{V}(T)=0.6 \exp \left(-4.03 \mathrm{eV} / k_{B} T\right) \mathrm{cm}^{2} \mathrm{~s}^{-1} .
$$

Both data sets predict a self-diffusion dominated by selfinterstitial (vacancy) mobility at a high (low) temperature. However, the crossover temperature for the dominating mechanism is different: $890{ }^{\circ} \mathrm{C}$ and $1000-1100{ }^{\circ} \mathrm{C}$, for Eqs. (3),(4) and (5),(6), respectively.

As for theory, a thorough study of self-diffusion requires accurate free-energy calculations (aimed at predicting temperature-dependent equilibrium concentrations) and extensive diffusivity simulations (aimed at computing migration energies and diffusivity prefactors) both for $I$ and $V$ defects. Once the formation free energies $F_{I, V}^{f}=E_{I, V}^{f}$ $+T S_{I, V}^{f}$, as well as migration energies $E_{I, V}^{m}$ and diffusivity prefactors $d_{I, V}^{0}$ are known, Eq. (2) can be recast in the form

$$
\begin{aligned}
D_{S D}(T)= & d_{I}^{0} \exp \left(-\frac{E_{I}^{f}-T S_{I}^{f}}{k_{B} T}\right) \exp \left(-E_{I}^{m} / k_{B} T\right) \\
& +d_{V}^{0} \exp \left(-\frac{E_{V}^{f}-T S_{V}^{f}}{k_{B} T}\right) \exp \left(-E_{V}^{m} / k_{B} T\right),
\end{aligned}
$$

so that a direct theory vs experiment comparison is possible.

At present, quantitative free-energy calculations are still rare, due to the considerable computational demands of finite-temperature simulations: an accurate simulation including high-temperature anharmonic effects, as well as reliable quantum-mechanical description of the bond dynamics involved in defect formation and migration, is in fact a very demanding task. A model potential molecular dynamics (MD) simulation, based on the Stillinger-Weber (SW) potential, has been presented by Sinno et al. ${ }^{8}$ Although the proposed numbers define a self-contained picture, the overall reliability is questionable, due to the limitation of the SW 
potential, as confirmed by the direct comparison with experimental data reported below. A much more reliable picture has been found by Blöchl et al. ${ }^{9}$ by means of first-principles calculations. In that work, however, the huge computational workload prevented from evaluating the temperature dependence of the $I$ contribution. Furthermore, as shown in the following, room for improvement appears as far as vacancymediated diffusion is concerned. Finally, a semi-empirical tight-binding molecular dynamics (TBMD) investigation ${ }^{10}$ has provided a reliable picture for formation and migration of native defects, while entropic contributions were fitted on experimental data or guessed from previous investigations. Thus, although the agreement between those TBMD data and experimental data ${ }^{2,7}$ was good, the theoretical investigation lacked of internal consistency and, therefore, can hardly be considered as predictive.

The above scenario points out the need for a new, selfcontained, quantum mechanical and atomistic investigation on self-diffusion constants, aimed at characterizing independently the two major mechanisms of diffusion. This is the scope of the present work, where TBMD free-energy calculations have been performed in the framework of the TB representation by Kwon et al. ${ }^{11}$ and eventually combined with previous TBMD diffusion simulations. ${ }^{10}$

The thermodynamic integration (TI) method ${ }^{12}$ was adopted to evaluate $F_{I, V}^{f}$. According to the TI formalism, the free-energy difference $\Delta F$ between two systems has been obtained by integrating

$$
\Delta F=\int_{\lambda=0}^{\lambda=1}\left\langle\frac{\partial H(\lambda)}{\partial \lambda}\right)_{\lambda} d \lambda
$$

where $\lambda$ acts as disposable parameter coupling a given initial state $H_{0}$ (with a well-known free energy) to the target final state (with unknown free energy) $H_{1}: H(\lambda)=\lambda H_{1}+(1$ $-\lambda) H_{0}$. In this work the state of reference was chosen to be an Einstein crystal. During the MD simulations a massive Nose-Hoover chain was attached to each vibrational degree of freedom to keep the ensemble canonical. ${ }^{13}$ The target state was, in turn, the TBMD Si sample. The ensemble average appearing in Eq. (8) was performed during constant volume, constant-temperature simulations on a $64 \pm 1$ atom periodically repeated cell. The net force $\mathbf{f}_{\alpha}$ acting on the $\alpha$ th atom was defined as $\mathbf{f}_{\alpha}=\lambda \mathbf{f}_{\alpha}^{T B M D}+(1-\lambda) \mathbf{f}_{\alpha}^{\text {Einst. }}$. At each statedefined by a given value of $\lambda$-the lattice was first carefully equilibrated with runs long up to $16 \mathrm{ps}$ (one time step corresponding to $1 \mathrm{fs}$ ) and finally averaged over several more ps. The thermodynamical integration was performed over $16 \lambda$ points. Our benchmarks (performed on 216 \pm 1 atoms and/or $\lambda=5$ points) proved that it is more important to include a higher number of $\lambda$ points in the TI evaluation than to enlarge the size of the simulation box. We estimate the present energy integrals given in Eq. (8) to be converged to within $\sim 10^{-4} \mathrm{eV}$. All free-energy calculations were performed at four different temperatures: $300,500,1000$, and $1400 \mathrm{~K}$.

The formation entropy $S_{I}^{f}$ for the self-interstitial defect was found almost constant with temperature, the average value being $S_{I}^{f}=11.2 k_{B}$. First-principles calculations performed in the local harmonic approximation ${ }^{9}$ predict a value of $S_{I}^{f} \sim 6 k_{B}$, which, however, increases up to $S_{I}^{f} \sim 10 k_{B}$ when including anharmonic terms through TI calculations. ${ }^{14}$ The case of a vacancy is more complicated. As a matter of fact, due to the high mobility of such a defect, ${ }^{10}$ during the equilibration + observation runs, the defect was mobile, thus adding a sizeable contribution of migration entropy in the TI free energy calculations. This is confirmed by the fact that the computed value of $S_{V}^{f}$ varied in the range $10.2-11.7 k_{B}$ in the selected temperature interval, with an average value of $S_{V}^{f}$ $\sim 10.8 k_{B}$. In fact, the migration contribution is already effectively included in TBMD simulations aimed at measuring $d_{V}^{0}$. To prevent the double counting of this term, we performed short observation runs, taking care to select only those simulations where $V$ diffusion actually did not take place. The convergence error thus included in our ensemble averages results in an entropy overestimation of $\sim 2 k_{B}$. In conclusion, we estimate an average formation (i.e., configurational+vibrational) entropy of $S_{V}^{f} \sim 8.8 k_{B}$. Our TBMD results for $S_{I}^{f}$ and $S_{V}^{f}$ are in good qualitative agreement with first-principles calculations by Blöchl et al. ${ }^{9,14}$ in the sense that the difference in the entropies of formation for $I$ 's and $V$ 's is of the order of $1-2 k_{B}$ in both studies, which predict a larger formation entropy for the interstitial.

Diffusivity constants were obtained by using the migration prefactors and energies computed in a previous work ${ }^{10}$ by means of the same TBMD scheme adopted here. In particular, we obtain $d_{I}^{0}=1.58 \times 10^{-1} \mathrm{~cm}^{2} \mathrm{~s}^{-1}$ and $d_{V}^{0}=1.18$ $\times 10^{-4} \mathrm{~cm}^{2} \mathrm{~s}^{-1}, E_{I}^{m}=1.37 \mathrm{eV}$ and $E_{V}^{m}=0.1 \mathrm{eV}$. As for the formation energies the TBMD results are $E_{I}^{f}=3.80 \mathrm{eV}$ and $E_{V}^{f}=3.97 \mathrm{eV}$. In this way, we get a self-consistent set of numbers, characterizing the various physical observables relevant to self-diffusion.

The vacancy contribution to self-diffusion predicted by present calculations is reported in Fig. 1. Experimental data by Bracht $e t a l^{2}$ and Gösele et al., ${ }^{7}$ as well as other $\mathrm{SW}^{8}$ and first-principles ${ }^{9,14}$ theoretical results are shown for comparison.

It is apparent that our TBMD investigation is in excellent agreement with state-of-the-art experimental data, providing

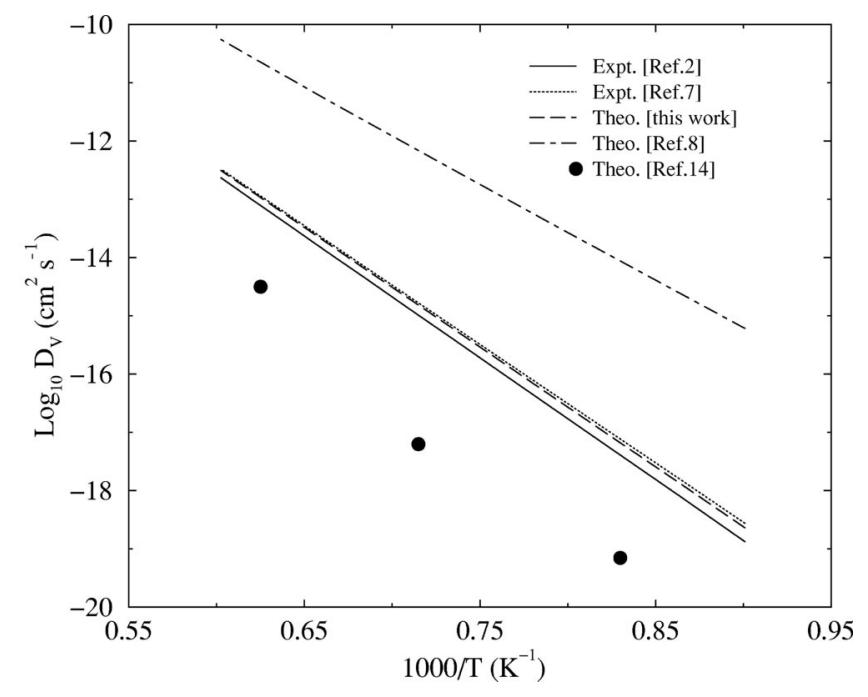

FIG. 1. Vacancy contribution to self-diffusion coefficient. 


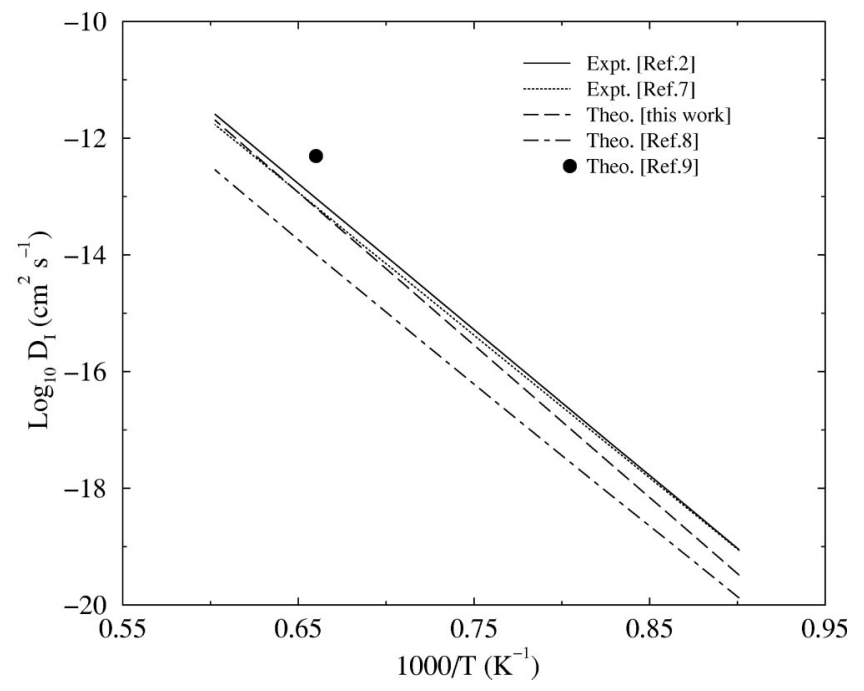

FIG. 2. Interstitial contribution to self-diffusion coefficient.

an overall atomistic picture for $V$-mediated self-diffusion much more reliable than model-potential simulations. We believe that the main source of disagreement between the SW and experimental data is due to the very low value of the vacancy formation energy $E_{V}^{f}=2.5 \mathrm{eV}$, which unfavorably compares with the $3.8 \mathrm{eV}$ and 3.3-3.7 eV values given by TBMD and first-principles calculations, respectively. ${ }^{10}$ The agreement is better for TBMD even when compared with $a b$ initio simulations which are principle superior. We guess that this is due to technical details of the calculations, both performed within the same formal framework, but with a different choice for the number of $\lambda$ points over which operate the TI integration (only three points were used in Ref. 14). Under this respect, the semi-empirical character of TBMD has proved to be an advantage: thanks to the reduced computational workload it is possible to perform TI simulations in a fully-converged regime, still keeping a quantum-mechanical description of atom interactions.

As for self-interstitials, Fig. 2 confirms the predictivity and reliability of the present results. We note that in this case model-potential data are not so bad, while first-principles

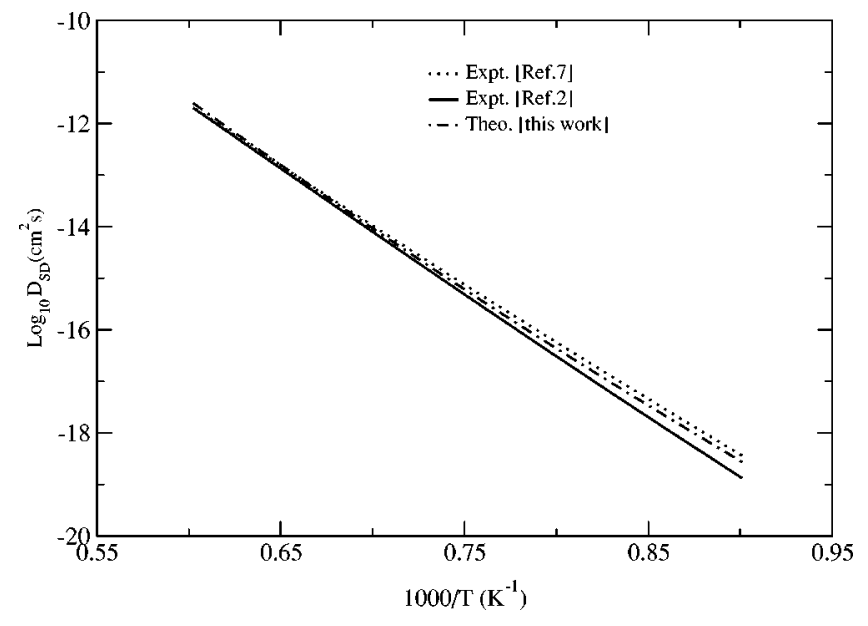

FIG. 3. Total self-diffusion coefficient in silicon.

calculations were performed at one temperature only, so that no estimation for the activation energy of $I$ diffusion can be obtained.

Finally, we remark that our investigation predicts the $I$ defects to dominate self-diffusion at high temperatures, as proved experimentally and confirmed by first-principles calculations. Furthermore, the $V$-mediated and $I$-mediated mechanisms give equal contributions at $\bar{T} \sim 1030^{\circ} \mathrm{C}$. This result is again consistent with available experiments. ${ }^{2,7}$

In conclusion, we propose the set of TBMD values reported here for the diffusion constants (which frequently may individually carry some model-related or method-related error) as the more complete and consistent set of values providing a correct global picture for self-diffusion in bulk Si. The degree of reliability of present results is summarized in Fig. 3 where the silicon TBMD total self-diffusion coefficient $D_{S D}=D_{I}+D_{V}$ is compared with state-of-the-art experimental data. ${ }^{2,7}$ The present numbers should be, therefore, useful in modeling $\mathrm{Si}$ bulk processing.

One of us (L.C.) acknowledges support by CNR under project "5\%-Microelettronica." This research has been supported by Academy of Finland through its Center of Excellence Program 2000-2006.
*Corresponding author.

Email address: luciano.colombo@dsf.unica.it

${ }^{1}$ W. Frank, U. Gösele, H. Meher, and A. Seeger, in Diffusion in Crystalline Solids, edited by G. E. Murch and A. Nowick (Academic Press, New York, 1984), p. 31.

${ }^{2}$ H. Bracht, E.E. Haller, and R. Clark-Phelps, Phys. Rev. Lett. 81, 393 (1998).

${ }^{3}$ K.C. Pandey, Phys. Rev. Lett. 57, 2287 (1986).

${ }^{4}$ K.C. Pandey and E. Kaxiras, Phys. Rev. Lett. 66, 915 (1990).

${ }^{5}$ K. Compaan and Y. Have, Trans. Faraday Soc. 52, 786 (1956); ibid. 54, 1498 (1958).

${ }^{6}$ H. Bracht, N.A. Stolwijk, and H. Mehrer, Phys. Rev. B 52, 16542 (1995).

${ }^{7}$ T.Y. Tan and U. Gösele, Appl. Phys. A: Solids Surf. 37, 1 (1985).
${ }^{8}$ T. Sinno, Z.K. Jiang, and R.A. Brown, Appl. Phys. Lett. 68, 3028 (1996).

${ }^{9}$ P.E. Blöchl, E. Smargiassi, R. Car, D.B. Lacks, W. Andreoni, and S.T. Pantelides, Phys. Rev. Lett. 70, 2435 (1993).

${ }^{10}$ M. Tang, L. Colombo, J. Zhu, and T. Diaz de la Rubia, Phys. Rev. B 55, 14279 (1997).

${ }^{11}$ I. Kwon, R. Biswas, C.Z. Wang, C.T. Chan, K.M. Ho, and C.M. Soukoulis, Phys. Rev. B 49, 4242 (1994).

${ }^{12}$ D. Frenkel and B. Smit, Understanding Molecular Simulations (Academic Press, New York, 1996).

${ }^{13}$ G.J. Martyna, M.L. Klein, and M. Tuckerman, J. Chem. Phys. 97, 2635 (1992).

${ }^{14}$ R. Car, P. Blöchl, and E. Smargiassi, Mater. Sci. Forum 83-87, 433 (1992) 